\title{
Toward AUV Survey Design for Optimal Coverage and Localization using the Cramer Rao Lower Bound
}

\author{
Ayoung Kim* and Ryan M. Eustice ${ }^{\dagger}$ \\ *Department of Mechanical Engineering \\ ${ }^{\dagger}$ Department of Naval Architecture \& Marine Engineering \\ University of Michigan \\ Ann Arbor, Michigan 48109-2145 \\ email:\{ayoungk, eustice\}@umich.edu
}

\begin{abstract}
This paper discusses an approach to using the Cramer Rao Lower Bound (CRLB) as a trajectory design tool for autonomous underwater vehicle (AUV) visual navigation. We begin with a discussion of Fisher Information as a measure of the lower bound of uncertainty in a simultaneous localization and mapping (SLAM) pose-graph. Treating the AUV trajectory as an non-random parameter, the Fisher information is calculated from the CRLB derivation, and depends only upon path geometry and sensor noise. The effect of the trajectory design parameters are evaluated by calculating the CRLB with different parameter sets. Next, optimal survey parameters are selected to improve the overall coverage rate while maintaining an acceptable level of localization precision for a fixed number of pose samples. The utility of the CRLB as a design tool in pre-planning an AUV survey is demonstrated using a synthetic data set for a boustrophedon survey. In this demonstration, we compare the CRLB of the improved survey plan with that of an actual previous hull-inspection survey plan of the USS Saratoga. Survey optimality is evaluated by measuring the overall coverage area and CRLB localization precision for a fixed number of nodes in the graph. We also examine how to exploit prior knowledge of environmental feature distribution in the survey plan.
\end{abstract}

\section{INTRODUCTION}

Recent developments in autonomous navigation have expanded autonomous underwater vehicle (AUV) applications to such areas as automated ship hull inspection [1], [2], autonomous underwater cave exploration [3], mapping of archaeological sites [4], [5], underwater exploration by tracking chemical plumes [6], and surveys of the underwater environment [7]-[9]. For many AUV missions, a typical survey criterion is to cover the largest survey area possible while satisfying a user-defined level of acceptable navigation performance. However, a physical limiting constraint in AUV mission duration is that they are governed by the amount of battery power that can be carried. Therefore, due to this power limitation, an optimal path and an efficient survey plan become a key aspect of the mission planning.

There are numerous examples in the literature for online path planning for AUVs; for example, obstacle avoidance using sonar sensing [10] and optimized trajectories for energy efficiency in the presence of water currents [11], [12]. What distinguishes our work is that what we are proposing is not an online method but rather a planning method to be used as a design tool for operator mission planning-allowing the AUV operator to make an informed trade off in coverage rate

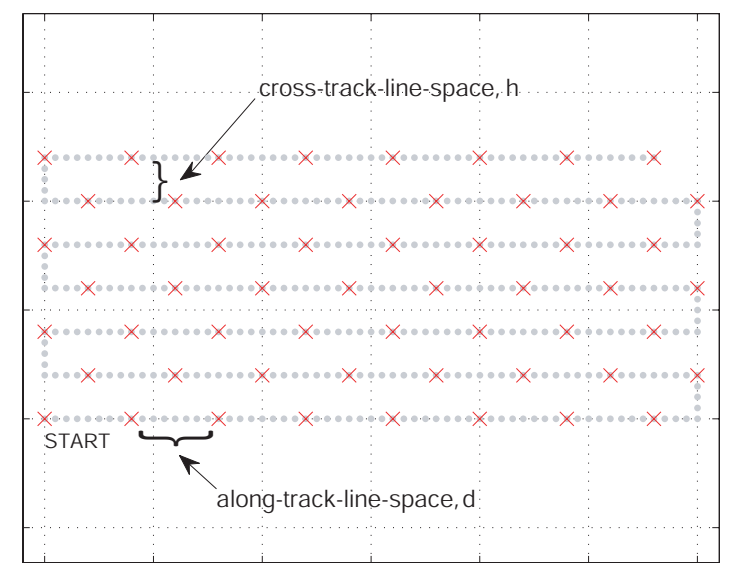

Fig. 1. A boustrophedon survey trajectory and its design parameters. The along-trackline-space, $d$, and cross-trackline-space, $h$, determine the posegraph node sampling, denoted as an $\mathrm{x}$, in the graph. The nodes, for example, could represent trajectory samples when optical imagery or sonar data along the hull are collected.

and localization precision for the typical boustrophedon survey pattern. In our application, these relative-pose constraints are derived from a visual simultaneous localization and mapping (SLAM) system [13]; however, they could as equally come from Iterated Closest Point (ICP) constraints derived from sonar [14] or laser [15] scan matching. In this paper, we define a performance metric based upon information gain as a measure of the survey's uncertainty level, and subsequently use this metric to provide optimal trajectory parameters in the design phase.

\section{A. Related Work}

A recent focus area in the robotics community has been to use information gain as a measure of uncertainty in order to improve the navigation and mapping accuracy of mobile platforms. Typically, information gain refers to either Fisher Information or Mutual Information, where the Fisher information matrix is closely related to the Cramer Rao Lower Bound (CRLB) [16], and Mutual information is defined from entropy [17]. In [18], Bingham introduced an approach for AUV navigation design using the CRLB in the survey design phase. In [19], Dogancay used the CRLB to investigate the design of optimal AOA (Angle of Arrival) for Unmanned 
Aerial Vehicle (UAV) tracking. In [20], Sim used the trace of the Fisher information matrix to determine the information gain in pose-graph SLAM. This direction of research was further developed in [21], where Vidal-Calleja's developed two different strategies related to information gain measures together with Mutual information gain.

\section{B. Motivation}

In our previous work, we implemented a vision-based SLAM algorithm, using 1,000 images to map a portion of the hull of the USS Saratoga aircraft carrier (Fig. 2 and Fig. 9). This work was done under the auspices of developing an AUV application for autonomous ship hull inspection [13]. The survey was designed to follow a boustrophedon trajectory along the hull using a pre-determined along-trackline-space, $d$, and cross-trackline-space, $h$ (Fig. 1). The survey track line distances were determined ahead of time by the AUV operators based upon the desired percentage overlap in the imagery, which was naively calculated from the expected vehicle position without taking into account the pose sampling effect on vehicle trajectory uncertainty. The hull-looking camera was set to operate at a fixed frame rate throughout the survey without any consideration to energy expenditure with each strobed image capture. In retrospect, the previous survey could have been more efficient with consideration to the amount of covered survey area, energy efficiency, and desired precision of localization performance had these criteria been simultaneously considered in the survey design phase.

\section{Outline}

Toward a solution to the optimal trajectory design problem, this paper examines using the CRLB as a metric for evaluating the effect of cross-trackline-spacing, $d$, and along-tracklinespacing, $h$, on the resulting boustrophedon pose-graph uncertainty. In $\S$ II we begin with a derivation of the CRLB assuming a standard sensor combination for AUVs, and introduce a metric to measure the graph uncertainty level. We illustrate the utility of this metric for a simple example case and explain its interpretation as a planning tool. In $\S$ III we then apply this design tool to the ship hull survey motivation example, and a comparison between the actual survey and the CRLB improved one is made. Finally, in $\S I V$ we offer some concluding remarks regarding what directions future work should take to improve this design tool.

\section{Cramer Rao Lower Bound}

We assume the AUV moves in a planar boustrophedon trajectory with a camera, a compass, and an odometry sensor (e.g., integrated Doppler velocity $\log (\mathrm{DVL}))$. We use notation of $\mathbf{x}_{i}=\left[x_{i}, y_{i}\right]^{\top}$ for the position of the vehicle along the trajectory at sample time $i$, and $\theta_{i}$ for the heading angle of the robot, where $\mathcal{X}_{i}=\left[\mathbf{x}_{i}^{\top}, \theta_{i}\right]^{\top}$ indicates the state of the vehicle. We use $\mathbf{Z}$ to indicate the stacked vector of all sensor measurements assembled from camera $\mathbf{Z}_{c}$, odometry $\mathbf{Z}_{o}$, and compass $\mathbf{Z}_{h}$ measurements, and $\mathbf{X}$ to indicate the stacked vector of all trajectory samples $\mathcal{X}_{i}$.

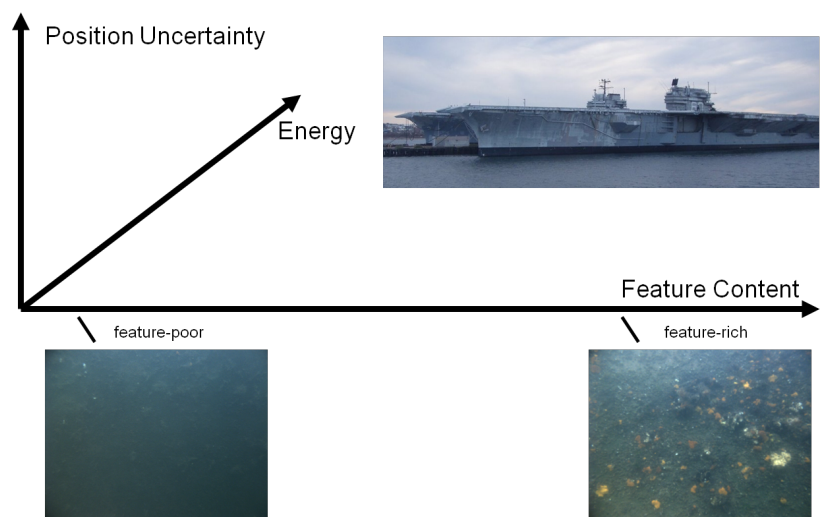

Fig. 2. Competing objectives in AUV mission planning. The desired level of position uncertainty, energy efficiency, and available feature content in the environment all impact the optimal SLAM trajectory design.

We use the CRLB (see [16] for standard reference)

$$
\mathrm{E}\left[\left[\hat{\mathbf{X}}(\mathbf{Z})-\mathbf{X}_{0}\right]\left[\hat{\mathbf{X}}(\mathbf{Z})-\mathbf{X}_{0}\right]^{\top}\right] \geq \mathrm{J}^{-1}
$$

as a measure of the conservative uncertainty bound for the pose-graph where $J$ in this equation is the Fisher information matrix

$$
\mathrm{J}=\left.\mathrm{E}\left[\left[\nabla_{\mathbf{X}} \ln \Lambda(\mathbf{X})\right]\left[\nabla_{\mathbf{X}} \ln \Lambda(\mathbf{X})\right]^{\top}\right]\right|_{\mathbf{X}_{0}}
$$

for the measurement likelihood $\Lambda(\mathbf{X})=p(\mathbf{Z} \mid \mathbf{X})$.

When we assume that all sensor measurements are independent, then the likelihood factorizes to a simple product whom after which taking its logarithm becomes a summation of all sensor log-likelihoods

$$
\begin{aligned}
\Lambda(\mathbf{X}) & =p(\mathbf{Z} \mid \mathbf{X}) \\
& =p\left(\mathbf{Z}_{c} \mid \mathbf{X}\right) p\left(\mathbf{Z}_{o} \mid \mathbf{X}\right) p\left(\mathbf{Z}_{h} \mid \mathbf{X}\right) \\
\ln \Lambda(\mathbf{X}) & =\ln p\left(\mathbf{Z}_{c} \mid \mathbf{X}\right)+\ln p\left(\mathbf{Z}_{o} \mid \mathbf{X}\right)+\ln p\left(\mathbf{Z}_{h} \mid \mathbf{X}\right) .
\end{aligned}
$$

We model the sensor measurements as corrupted by Gaussian noise with zero mean and covariances $\Sigma_{c_{i j}}, \Sigma_{o_{i}}$, and $\sigma_{h_{i}}^{2}$, respectively:

Camera: $\quad \mathbf{Z}_{c_{i j}}=\mathbf{g}\left(\mathcal{X}_{i}, \mathcal{X}_{j}\right)+\mathbf{W}_{c_{i j}}, \mathbf{W}_{c_{i j}} \sim \mathcal{N}\left(0, \Sigma_{c_{i j}}\right)$, Odometry: $\quad \mathbf{Z}_{o_{i}}=\left(\mathbf{x}_{i}-\mathbf{x}_{i-1}\right)+\mathbf{W}_{o_{i}}, \mathbf{W}_{o_{i}} \sim \mathcal{N}\left(0, \Sigma_{o_{i}}\right)$, Compass: $\quad Z_{h_{i}}=\theta_{i}+W_{h_{i}}, W_{h_{i}} \sim \mathcal{N}\left(0, \sigma_{h_{i}}^{2}\right)$,

where we assume a standard deviation of $\pm 1.2 \mathrm{~cm} / \mathrm{s}$ in DVL velocity in computing the integrated odometry measurement [22] and a fixed $\pm 1^{\circ}$ for heading uncertainty (i.e., $\sigma_{h_{i}}=\sigma_{h}=$ $\left.\pm 1^{\circ}\right)$.

\section{A. Modeling of the Camera Measurement}

For the camera measurement we use a reduced two degree of freedom (DOF) camera measurement model $\mathrm{g}\left(\boldsymbol{\mathcal { X }}_{i}, \boldsymbol{\mathcal { X }}_{j}\right)$ for the planar survey, which measures the azimuth, $\alpha_{i j}$, and relative orientation, $\beta_{i j}$, between nodes $i$ and $j$, which models 


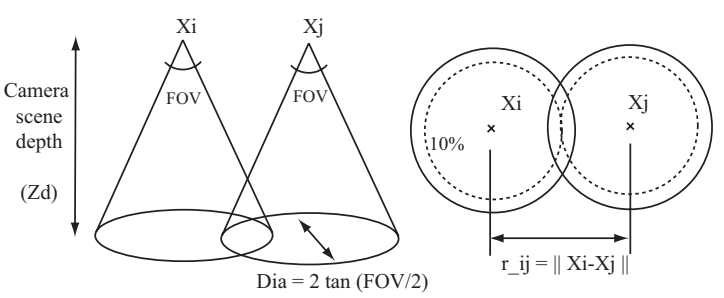

(a) Pairwise overlap model of camera measurement

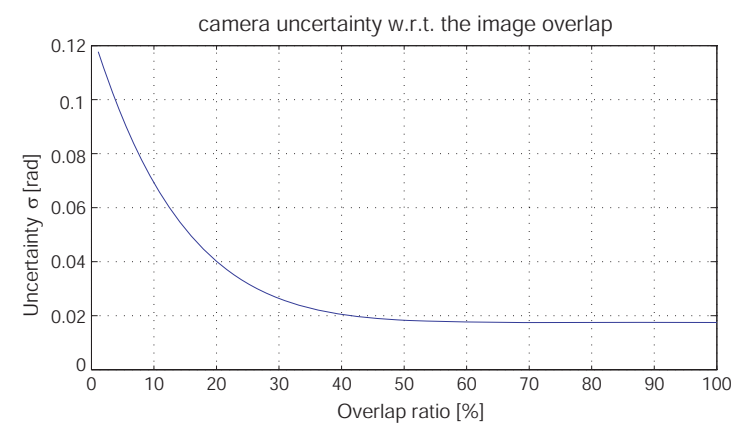

(b) Standard deviation of the camera measurement noise as a function of the overlap ratio

Fig. 3. Camera measurement noise is modeled as a function of the image overlap ratio from the pairwise camera measurement.

the camera as a bearing sensor making both sequential and non-sequential links in the graph.

$$
\mathbf{g}\left(\mathcal{X}_{i}, \mathcal{X}_{j}\right)=\left[\begin{array}{c}
\alpha_{i j} \\
\beta_{i j}
\end{array}\right]=\left[\begin{array}{c}
\operatorname{atan} 2\left(y_{j}-y_{i}, x_{j}-x_{i}\right) \\
\theta_{j}-\theta_{i}
\end{array}\right]
$$

The camera sensor measurement noise is modeled so as to be dependent on the distance between two nodes where the camera measurement uncertainty $\Sigma_{c_{i j}}$ is modeled as a function of the overlap ratio. The size of the overlap region is commensurate with the proximity of node $i$ and $j$; the closer nodes $i$ and $j$, the larger the overlap region, resulting in less uncertainty in the camera measurement. As can been seen from Fig 3(a), with a fixed camera field of view (FOV) and the camera scene depth $z_{d}$, the overlap ratio becomes a function of the distance of two nodes only. This fixed scene depth assumption is for the survey of the bottom or the wall following. For simplicity, we assume the footprint of the camera measurement to be a circle as seen in Fig 3(a), and define camera measurement threshold $C_{\text {thresh }}=2 z_{d} \tan (\mathrm{FOV} / 2)$ and the overlap ratio as $\gamma=\frac{\left\|X_{i}-X_{j}\right\|}{C_{\text {thresh }}}$. Only when the distance $r_{i j}$ is smaller than $C_{t h r e s h}$, is the camera measurement meaningful and the information gain is proportional to the distance $r_{i j}$. The function that defines the uncertainty of the camera measurement

$$
\sigma= \pm 1^{\circ} \gamma^{5}
$$

is shown in Fig. 3(b). When the overlap ratio falls below 10\%, the uncertainty increases significantly, and for greater than $50 \%$ it remains relatively flat. We assume the camera FOV to be $40^{\circ}$, which gives the $C_{t h r e s h}$ to be $0.7279 \mathrm{~m}$ at the scene depth of $1 \mathrm{~m}$, for example.

\section{B. Fisher Information Matrix}

As previously shown in (2), the Fisher Information matrix, J, is simply the expectation of the squared gradient of the log-likelihood evaluated at the true parameters $\mathbf{X}_{0}$. Using the assumption of sensor measurement independence, the expectation of the gradient from two different sensor measurement likelihoods is zero (i.e., $\mathrm{E}\left[\left[\nabla_{\mathbf{X}} \ln p\left(\mathbf{Z}_{m} \mid \mathbf{X}\right)\right]\left[\nabla_{\mathbf{X}} \ln p\left(\mathbf{Z}_{n} \mid \mathbf{X}\right)\right]^{\top}\right]=0$ when $\left.m \neq n\right)$. Therefore, J reduces to the three simple sensor terms below

$$
\begin{aligned}
\mathrm{J} & =\mathrm{E}\left[\left[\nabla_{\mathbf{X}} \ln p\left(\mathbf{Z}_{c} \mid \mathbf{X}\right)\right]\left[\nabla_{\mathbf{X}} \ln p\left(\mathbf{Z}_{c} \mid \mathbf{X}\right)\right]^{\top}\right] \\
& +\mathrm{E}\left[\left[\nabla_{\mathbf{X}} \ln p\left(\mathbf{Z}_{o} \mid \mathbf{X}\right)\right]\left[\nabla_{\mathbf{X}} \ln p\left(\mathbf{Z}_{o} \mid \mathbf{X}\right)\right]^{\top}\right] \\
& +\mathrm{E}\left[\left[\nabla_{\mathbf{X}} \ln p\left(\mathbf{Z}_{h} \mid \mathbf{X}\right)\right]\left[\nabla_{\mathbf{X}} \ln p\left(\mathbf{Z}_{h} \mid \mathbf{X}\right)\right]^{\top}\right] .
\end{aligned}
$$

By defining information matrices $\mathrm{L}_{c}, \mathrm{~L}_{o}$, and $\mathrm{L}_{h}$, to correspond to the three terms above, respectively, the Fisher information $\mathrm{J}$ can be written as the summation of the three information matrices as contributed from each sensor

$$
\mathrm{J}=\mathrm{L}_{\mathrm{o}}+\mathrm{L}_{\mathrm{h}}+\mathrm{L}_{\mathrm{c}} .
$$

The detailed general structure for the three information matrices is given in equations (6) and (7).

Unlike the odometry and heading sensors, the camera information matrix, $\mathrm{L}_{c}$, is a function of geometry and can be written in terms of the camera observation model gradient as

$$
\mathrm{D}_{i j}=\frac{\partial \mathbf{g}\left(\boldsymbol{\mathcal { X }}_{i}, \boldsymbol{\mathcal { X }}_{j}\right)}{\partial \mathcal{X}_{i}} \Sigma_{c_{i j}}^{-1} \frac{\partial \mathbf{g}\left(\boldsymbol{\mathcal { X }}_{i}, \boldsymbol{\mathcal { X }}_{j}\right)^{\top}}{\partial \mathcal{X}_{j}} .
$$

While the information matrix of the odometry and heading sensors depend only on the sensor uncertainty and integration interval, (5) makes it clear that the camera information is a function of the sensor uncertainty and the trajectory geometry. In particular, $\mathrm{L}_{c}$ will exhibit nonzero elements in the off diagonal in the Fisher information matrix as the camera measurement can result from non-sequential nodes in the graph.

\section{CRLB Derivation Example}

In this section, we examine a simple pose-graph consisting of three nodes as depicted in Fig. 4, and present a detailed calculation of the three sensor information matrices. In this example, two sequential nodes, $(1,2)$, and $(2,3)$, are constrained by the odometry sensor measurement. On each node, we have compass information, but we only have the camera measurement between nodes 2 and 3, which is depicted by the green line in Fig. 4. As we have three elements $x, y$, and $\theta$ in our state representation, the three information matrices will each be $9 \times 9$ matrices.

Under this scenario, $\mathrm{L}_{c}$ is calculated as

$$
\begin{aligned}
\mathrm{L}_{c} & =\left[\begin{array}{ccc}
\mathrm{D}_{12}+\mathrm{D}_{13} & -\mathrm{D}_{12} & -\mathrm{D}_{13} \\
-\mathrm{D}_{12} & \mathrm{D}_{12}+\mathrm{D}_{23} & -\mathrm{D}_{23} \\
-\mathrm{D}_{13} & -\mathrm{D}_{23} & \mathrm{D}_{13}+\mathrm{D}_{23}
\end{array}\right] \\
& =\left[\begin{array}{ccc}
0_{3 \times 3} & 0_{3 \times 3} & 0_{3 \times 3} \\
0_{3 \times 3} & \mathrm{D}_{23} & -\mathrm{D}_{23} \\
0_{3 \times 3} & -\mathrm{D}_{23} & \mathrm{D}_{23}
\end{array}\right]
\end{aligned}
$$




$$
\begin{aligned}
& \mathrm{L}_{o}+\mathrm{L}_{h}=\mathrm{E}\left[\left[\nabla_{\mathbf{X}} \ln p\left(\mathbf{Z}_{o} \mid \mathbf{X}\right)\right]\left[\nabla_{\mathbf{X}} \ln p\left(\mathbf{Z}_{o} \mid \mathbf{X}\right)\right]^{\top}\right]+\mathrm{E}\left[\left[\nabla_{\mathbf{X}} \ln p\left(\mathbf{Z}_{h} \mid \mathbf{X}\right)\right]\left[\nabla_{\mathbf{X}} \ln p\left(\mathbf{Z}_{h} \mid \mathbf{X}\right)\right]^{\top}\right]
\end{aligned}
$$

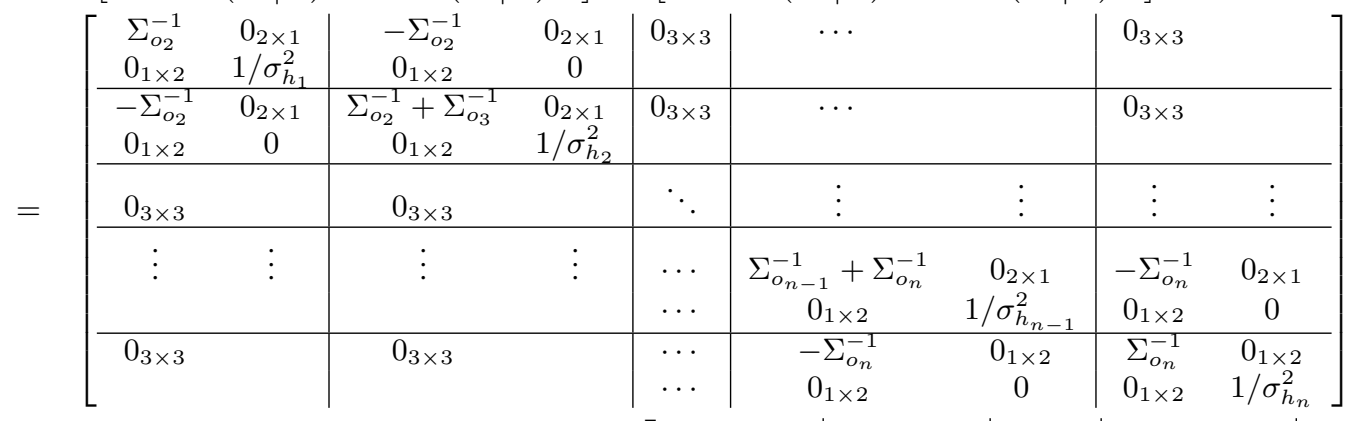

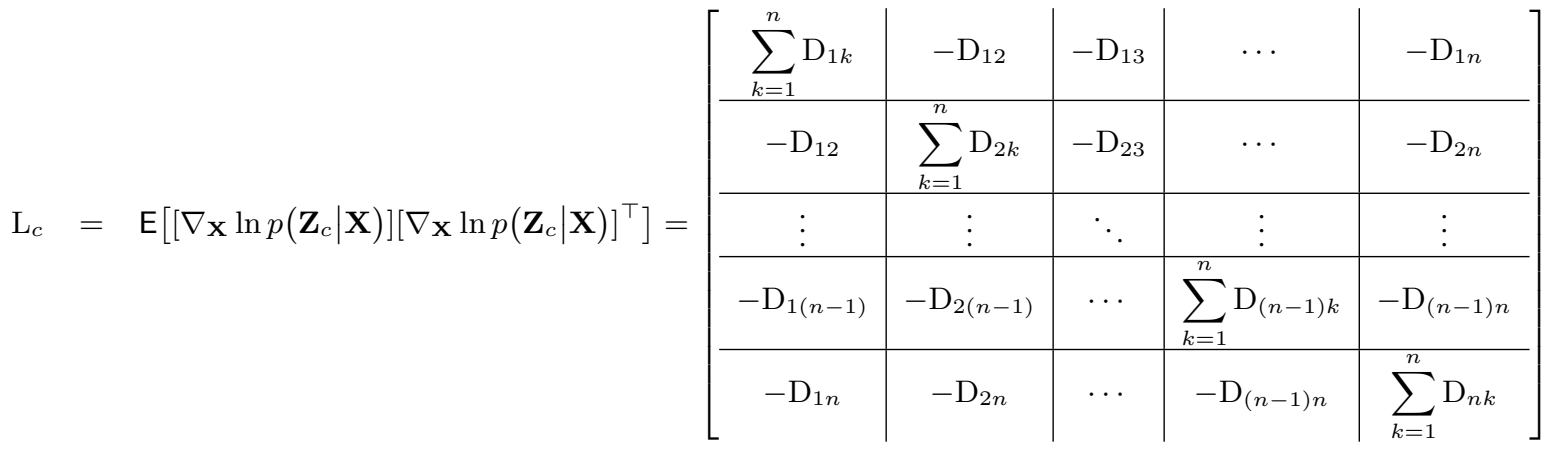

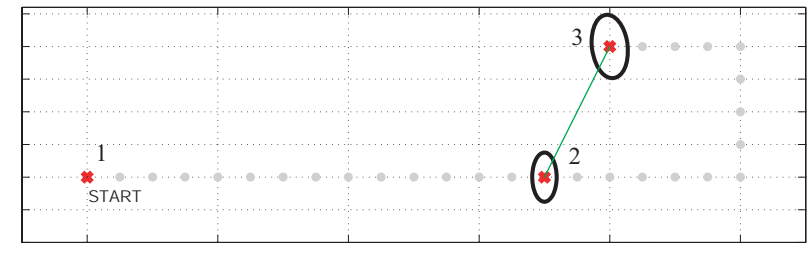

Fig. 4. A simple example of a three node graph to illustrate the derivation of the information matrices in the CRLB calculation.

and $\mathrm{L}_{o}+\mathrm{L}_{h}=$

$\left[\begin{array}{cc|cc|cc}\Sigma_{o_{2}}^{-1} & 0_{2 \times 1} & -\Sigma_{o_{2}}^{-1} & 0_{2 \times 1} & 0_{3 \times 3} & \\ 0_{1 \times 2} & 1 / \sigma_{h}^{2} & 0_{1 \times 2} & 0 & & \\ \hline-\Sigma_{o_{2}}^{-1} & 0_{2 \times 1} & \Sigma_{o_{2}}^{-1}+\Sigma_{o_{3}}^{-1} & 0_{2 \times 1} & -\Sigma_{o_{2}}^{-1} & 0_{2 \times 1} \\ 0_{1 \times 2} & 0 & 0_{1 \times 2} & 1 / \sigma_{h}^{2} & 0_{1 \times 2} & 0 \\ \hline 0_{3 \times 3} & & -\Sigma_{o_{2}}^{-1} & 0_{2 \times 1} & \Sigma_{o_{3}}^{-1} & 0_{2 \times 1} \\ & & 0_{1 \times 2} & 0 & 0_{2 \times 1} & 1 / \sigma_{h}^{2}\end{array}\right]$.

Summing all terms, the Fisher information matrix is calculated to be $\mathrm{J}=\mathrm{L}_{h}+\mathrm{L}_{o}+\mathrm{L}_{c}$, which is singular because it contains only relative-pose information (i.e. camera and odometry measurements are unaffected by a linear translation). Therefore, we fix the location of the first node in the graph by setting its information to be infinity (in practice a very large number like $10^{6}$ ), so that the other node uncertainties are relative to the first node position.

Taking the inverse of the resulting $\mathrm{J}$ matrix yields the $9 \times 9$ covariance matrix $\mathrm{S}$

$$
\mathrm{S}=\mathrm{J}^{-1}=\left[\begin{array}{lll}
\mathrm{S}_{11} & \mathrm{~S}_{12} & \mathrm{~S}_{13} \\
\mathrm{~S}_{12} & \mathrm{~S}_{22} & \mathrm{~S}_{23} \\
\mathrm{~S}_{13} & \mathrm{~S}_{23} & \mathrm{~S}_{33}
\end{array}\right]
$$

where $\mathrm{S}_{i i}$ indicates the covariance of the $i^{\text {th }}$ node and $\mathrm{S}_{i j}$ the $i^{t h}, j^{\text {th }}$ cross-covariance.

\section{Effect of the Design Parameters}

We examine the CRLB by evaluating the inverse of the Fisher Information matrix, which gives the covariance matrix, $\mathrm{S}$, of the overall graph. The determinant of each covariance matrix sub-block, $\mathrm{S}_{i i}$, reveals the uncertainty of each node in the graph. We evaluate the maximum $x y$ standard deviation for all nodes in the graph $\left(\max _{i} \sqrt[4]{\left|S_{x y_{i i}}\right|}\right)$ by looking at the $S_{x y}$ sub-block for each node and use this as a metric for the level of $x y$ uncertainty in the entire trajectory. Figures 5 and 6 depict this survey metric for a more extended case considering a 40 node trajectory.

With respect to the odometry sensor uncertainty on the $i^{t h}$ node, for example, $\Sigma_{o_{i}}$ will increase as the along-tracklinespace, $d$, increases because it accumulates DVL uncertainty over longer distances. On the other hand, the reduction of the cross-trackline-space, $h$, will enhance the overall pose-graph link structure as the camera can make a larger number of nonsequential links to other tracklines. In terms of the camera measurements, small $d$ and $h$ values enable a higher density of camera measurements, thereby reducing the uncertainty; however, this also reduces the size of the survey coverage area as depicted in plans A, and D of Fig. 5(a). Therefore we examine the relation of the uncertainty and the survey coverage with respect to the design parameters to achieve an optimal balance given these competing considerations.

The two graphs in Fig 5(b) show the relation of the camera measurement with the two independent design parameters $d$ and $h$. As can be seen in Fig. 5(b), the maximum pose 


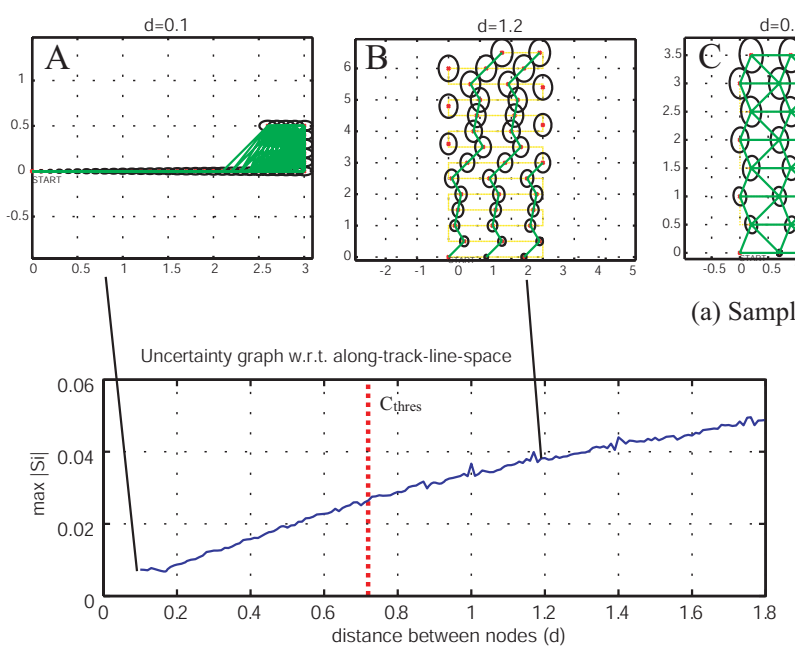

(b) Evaludated uncertainty w.r.t. $\mathrm{d}$ and $\mathrm{h}$

Fig. 5. Change of maximum uncertainty with respect to the altitude normalized along-trackline-space, $d$, and the cross-trackline-space, $h$. Example trajectory design paths are depicted in (a).

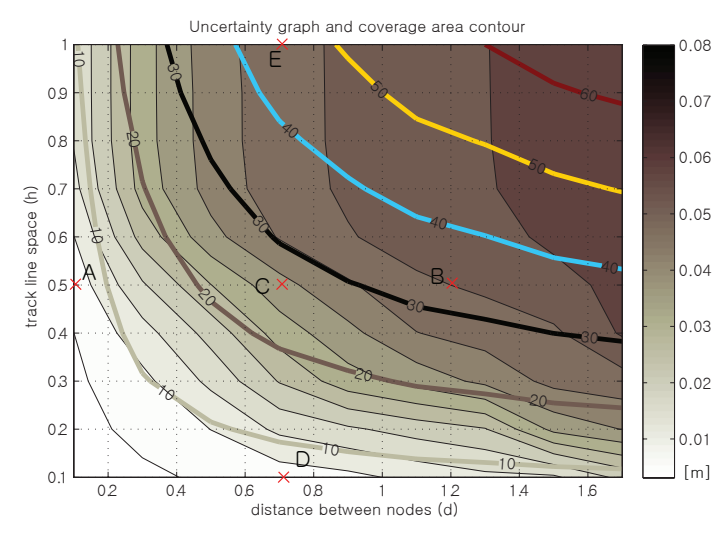

Fig. 6. The contour plot of the maximum uncertainty and the coverage area. The letters from A to E indicate each sample plan in Fig. 5. The curved lines with numbers indicate the altitude normalized coverage area of the plan, while the contours underneath show the altitude normalized maximum standard deviation from the CRLB. The colorbar on the left side represents the uncertainty level.

uncertainty does not grow rapidly until it passes the camera measurement threshold, $C_{\text {thresh }}$. Once the cross-tracklinespace exceeds that of the camera measurement threshold, it no longer makes cross-track links, which significantly increases the maximum trajectory uncertainty (e.g. plan E). Therefore, without considering the coverage area, it would be reasonable to select the value of $d$ and $h$ according to the camera measurement threshold, $C_{\text {thresh }}$, which is a function of the camera's intrinsic parameters, field of view, and scene altitude. However, since the uncertainty and coverage area should be evaluated simultaneously, a contour plot containing both factors is plotted as in Fig. 6, which can be used to analyze and optimally plan the survey.

In the contour plot, each sample path is marked with capital letters from A to E. Path $\mathrm{C}$ has been selected to be optimal in that it achieves the minimum uncertainty for coverage of the largest area among the parameters for the same uncertainty level. With plan $\mathrm{C}(d=0.7$ and $h=0.5)$, an altitude normalized max standard deviation of 0.017 is estimated for an altitude normalized total coverage area of 10.4.

\section{Simulation Result}

We examine our previous hull-inspection survey results of the USS Saratoga [13] using our newfound CRLB analysis, and look for possible improvements that we could have made to the original survey trajectory by analyzing the relationship between the CRLB and survey design parameters. We continue to use the maximum of the covariance matrix of all nodes in the graph as our uncertainty metric $\left(\max _{i} \sqrt[4]{\left|\mathrm{S}_{x y_{i i}}\right|}\right)$, which yields the contour plot depicted in Fig. $7^{i}$.

\section{A. Improvement in the Saratoga Survey}

The previous USS Saratoga survey was planned using along-trackline-space, $d$, and cross-trackline-space, $h$, which were calculated based upon the desired percentage of image overlap without taking into account any effect on pose uncertainty. The vehicle survey was configured for a constant distance from the ship hull, maintaining a standoff distance of $1.5 \mathrm{~m}$ from the hull. To maintain a cross-track image overlap percentage of $54 \%, h$ was calculated to be $0.5 \mathrm{~m}$. We did not intentionally control the along-trackline-space $d$, rather we set the camera to run at a fixed frame rate of $1 \mathrm{fps}$, which at a forward speed of $0.2 \mathrm{~m} / \mathrm{s}$ resulted in an along-track sample spacing of approximately $0.2 \mathrm{~m}$. With $d=0.2 \mathrm{~m}$ and $h=0.5 \mathrm{~m}$, a large along-track image overlap ratio of $81.68 \%$ was achieved for the overall survey. According to the present CRLB analysis, the minimum achievable uncertainty for this survey is calculated to be $0.0141 \mathrm{~m}$ using 1060 nodes to cover a $89.40 \mathrm{~m}^{2}$ area. This previous survey plan is denoted with the letter $\mathrm{G}$ in Fig. 7.

To calculate the optimal survey parameters, the uncertainty and the coverage area are evaluated with respect to different 


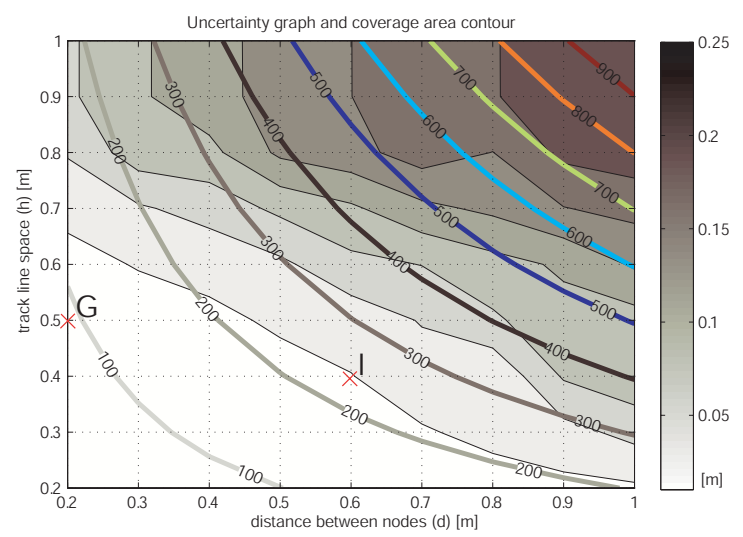

(a) Evaluation of the uncertainty level and the coverage area with respect to design parameters

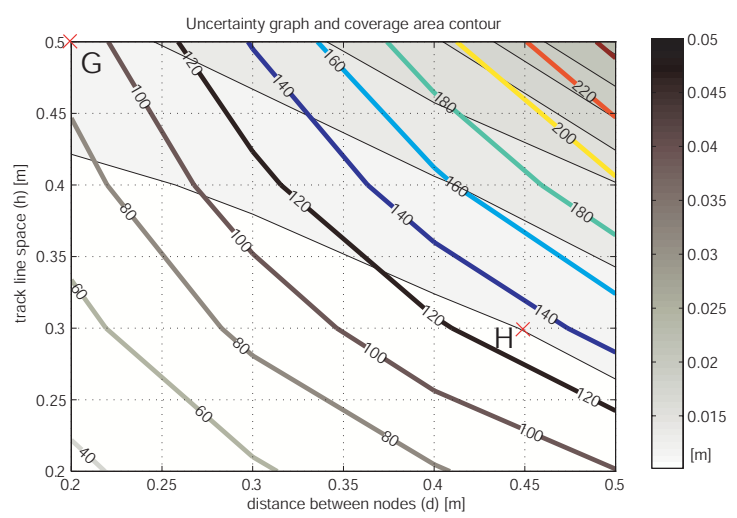

(b) Zoomed contour plot

Fig. 7. Parameter evaluation for saratoga data. Point $\mathrm{G}$ indicates the previous survey plan. We propose two improved plans marked as $\mathrm{H}$ and $\mathrm{I}$.

sets of the design parameters as in Fig. 7. Our goal is to find the set of parameters that covers the largest area with the lowest uncertainty. Here, by examining the contour plot of Fig. 7, we can find two sets of parameters that will improve the result, which are summarized in Table I below.

TABLE I

Optimal USS SaRatoga SuRVey PaRameters

\begin{tabular}{c|ccccc} 
Plan & $d(\mathrm{~m})$ & $h(\mathrm{~m})$ & $\max \sqrt[4]{\left|\mathrm{S}_{x y_{i i} \mid}\right|}(\mathrm{m})$ & Area $\left(\mathrm{m}^{2}\right)$ & \\
\hline $\mathrm{G}$ & 0.2 & 0.5 & 0.0141 & 89.4 & \\
$\mathrm{H}$ & 0.45 & 0.3 & 0.0103 & 132.6 & $(48.3 \%)$ \\
$\mathrm{I}$ & 0.5 & 0.6 & 0.0448 & 293.5 & $(228.3 \%)$
\end{tabular}

The first plan, $\mathrm{H}$, reduces the uncertainty while simultaneously increasing the coverage area. This strategy moves the original survey point $\mathrm{G}$ in the contour plot down toward the lowest uncertainty contour, then right toward the largest possible coverage area for that level of uncertainty. This plan provides $26.9 \%$ reduced uncertainty while covering $48.3 \%$ more area. On the other hand, considering the fact that the survey is already highly conservative and that the uncertainty is significantly small, we could accept the increase of uncertainty for the purpose of achieving a dramatically larger survey coverage. This strategy is illustrated by point I on the contour

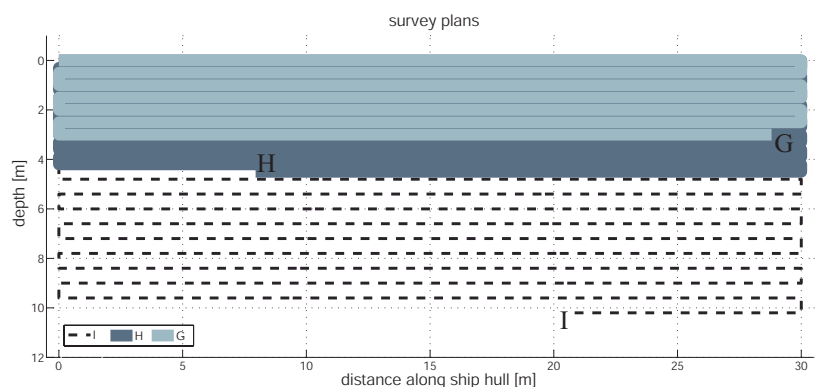

Fig. 8. Original and simulated trajectories for the two improved surveys.

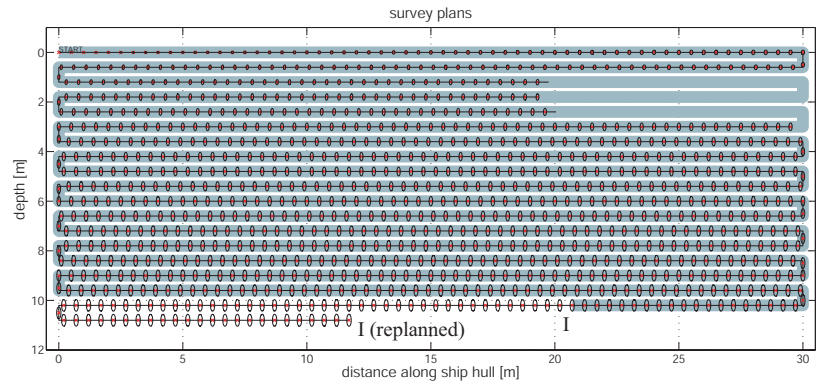

(a) Re-planned survey I by node removal

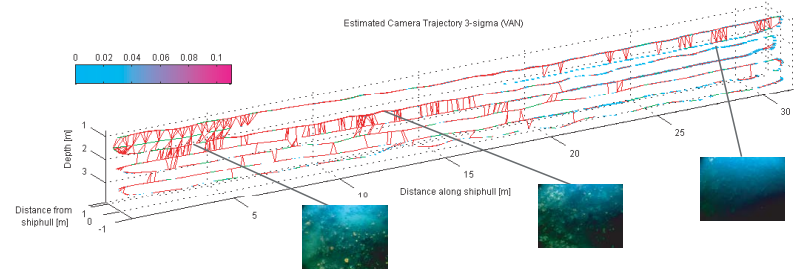

(b) Feature distribution information from the original survey

Fig. 9. The area without enough feature distribution is revealed from the previous survey. The improved survey plan is proposed as in (a). Ellipsoids at each node in (a) illustrates $1-\sigma$ contour of the position uncertainty, which are inflated by 10 for visibility.

graph where the uncertainty increases to $0.0448 \mathrm{~m}$, (but is still in absolute terms small) while covering $228.3 \%$ more area. The two sample paths generated from these two improved surveys are illustrated in Fig. 8.

\section{B. Exploiting Feature Distribution in the Survey Design}

The distribution of features and texture in the environment is a highly dominant factor in the SLAM sensor measurement quality. For example, for the vision system the distribution of features in the environment is critical to registration quality, and it will determine the performance of the camera measurement. However, real environments typically do not exhibit uniform feature distributions. There therefore exist areas in the survey where the measurement might not be reliable due to feature-less regions (e.g., sand areas, empty regions or areas of low texture). Hence, when we have information about the environment prior to our survey, or when we re-plan the same area with data from a previous survey, we can exploit this knowledge in our survey design in order to cover more area by reducing our pose sampling in any featureless areas. In the 
case of the ship hull survey, many featureless region exists and fail to provide relative-pose constraints. By removing nodes from those areas that are expected to be inefficient, we could use the saved energy from the removal of the nodes to conduct further exploration, despite the expense of temporarily increased uncertainty over that region.

The simulated result of the removal of nodes with the same design parameters of plan I (Fig. 8) is provided in Fig. 9. From the result of the previous survey in Fig. 9(b), we know that the area of $10 \mathrm{~m}$ by $2 \mathrm{~m}$ is a largely feature-less region, which is equivalent to 66 nodes in the graph. Therefore, as can be seen Fig. 9(a), these 66 nodes were removed from the middle section, extending the total path length but maintaining an uncertainty level equivalent to that in plan I.

\section{CONCLUSion}

This paper described a method for improving the localization performance of a pose-graph SLAM methodology using sequential/non-sequential relative-pose constraints. A conservative static uncertainty bound was examined by calculating the CRLB in the AUV survey design phase, and thereby determining the uncertainty level of the graph. A graphical design tool based upon contour plots of coverage area and maximum uncertainty was developed to interpret the effects of the design parameters on the survey coverage and uncertainty. Using this tool, design parameters were proposed to meet the purpose of the survey as a pre-planning instrument. A method to exploit the environmental information for use in the survey was also proposed.

In this preliminary result, we assumed a fixed number of nodes in the graph and found the two design parameters for a simple boustrophedon trajectory. Future work will include modeling additional terms important to the survey design, such as the total amount of time of the mission or the total energy limitation of the vehicle. The total number of nodes in the graph should be variable as a function of the amount of energy the vehicle can expend and the overall trajectory path length. Similar contour plots could be generated with this information on it, and we could use the same graphical method to optimize the survey in the pre-planning phase.

\section{ACKNOWLEDGMENTS}

This work is supported in part through a grant from the Office of Naval Research (ONR) (Award \#N00014-07-1-0791) and the National Science Foundation (NSF) (Award \#IIS 0746455).

\section{REFERENCES}

[1] H. C. Brown, A. Kim, and R. M. Eustice, "An overview of autonomous underwater vehicle research and testbed at PeRL," Marine Technology Society Journal, vol. 43, no. 2, pp. 33-47, 2009.

[2] J. Vaganay, M. Elkins, S. Willcox, F. Hover, R. Damus, S. Desset, J. Morash, and V. Polidoro, "Ship hull inspection by hull-relative navigation and control," OCEANS, 2005. Proceedings of MTS/IEEE, pp. 761-766 Vol. 1, 2005.

[3] N. Fairfield, G. A. Kantor, D. Jonak, and D. Wettergreen, "DEPTHX autonomy software: design and field results," Robotics Institute, Carnegie Mellon University, Pittsburgh, PA, Tech. Rep. CMU-RI-TR-08-09, July 2008.
[4] R. M. Eustice, H. Singh, J. J. Leonard, and M. R. Walter, "Visually mapping the RMS Titanic: conservative covariance estimates for SLAM information filters," International Journal of Robotics Research, vol. 25, no. 12, pp. 1223-1242, 2006.

[5] H. Singh, J. Howland, and O. Pizarro, "Advances in large-area photomosaicking underwater," IEEE Journal of Oceanic Engineering, vol. 29, no. 3, pp. 872-886, Jul. 2004.

[6] J. Farrell, S. Pang, and W. Li, "Chemical plume tracing via an autonomous underwater vehicle," Oceanic Engineering, IEEE Journal of, vol. 30, no. 2, pp. 428-442, April 2005.

[7] R. Armstrong, H. Singh, J. Torres, R. Nemeth, A. Can, C. Roman, R. Eustice, L. Riggs, and G. Garcia-Moliner, "Characterizing the deep insular shelf coral reef habitat of the Hind Bank marine conservation district (US Virgin Islands) using the Seabed autonomous underwater vehicle," Continental Shelf Research, vol. 26, no. 2, pp. 194-205, Feb. 2006.

[8] D. Yoerger, A. Bradley, H. Singh, B. Walden, M. Cormier, and W. Ryan, "Multisensor mapping of the deep seafloor with the Autonomous Benthic Explorer," in Proceedings of the International Symposium on Unmanned Untethered Submersible Technology, Tokyo, Japan, May 2000, pp. 248253.

[9] O. Pizarro, R. Eustice, and H. Singh, "Large area 3D reconstructions from underwater surveys," in Proceedings of the IEEE/MTS OCEANS Conference and Exhibition, vol. 2, Kobe, Japan, Nov. 2004, pp. 678-687.

[10] Y. Petillot, I. Tena Ruiz, and D. Lane, "Underwater vehicle obstacle avoidance and path planning using a multi-beam forward looking sonar,' IEEE Journal of Oceanic Engineering, vol. 26, no. 2, pp. 240-251, 2001.

[11] D. Kruger, R. Stolkin, A. Blum, and J. Briganti, "Optimal AUV path planning for extended missions in complex, fast-flowing estuarine environments," in ICRA, 2007, pp. 4265-4270.

[12] K. Carroll, S. McClaran, E. Nelson, D. Barnett, D. Friesen, and G. William, "Auv path planning: an a* approach to path planning with consideration of variable vehicle speeds and multiple, overlapping, timedependent exclusion zones," Autonomous Underwater Vehicle Technology, 1992. AUV '92., Proceedings of the 1992 Symposium on, pp. 79-84, Jun 1992.

[13] A. Kim and R. M. Eustice, "Pose-graph visual SLAM with geometric model selection for autonomous underwater ship hull inspection," in Proceedings of the IEEE/RSJ International Conference on Intelligent Robots and Systems, 2009, To Appear.

[14] C. Roman and H. Singh, "Improved vehicle based multibeam bathymetry using sub-maps and SLAM," in Proceedings of the IEEE/RSJ International Conference on Intelligent Robots and Systems, Edmonton, Alberta, Canada, Aug. 2005, pp. 2422-2429.

[15] E. Olson, "Real-time correlative scan matching," in Proceedings of the IEEE International Conference on Robotics and Automation (ICRA), Kobe, Japan, June 2009, pp. 4387-4393.

[16] Y. Bar-Shalom, X. Rong Li, and T. Kirubarajan, Estimation with applications to tracking and navigation. New York: John Wiley \& Sons, Inc., 2001.

[17] M. Bryson and S. Sukkarieh, "An information-theoretic approach to autonomous navigation and guidance of an uninhabited aerial vehicle in unknown environments," in Intelligent Robots and Systems, 2005. (IROS 2005). 2005 IEEE/RSJ International Conference on, Aug. 2005, pp. 3770-3775.

[18] B. Bingham, "Precision autonomous underwater navigation," Ph.D. dissertation, Massachusetts Institute of Technology, May 2003.

[19] K. Doğançay and H. Hmam, "Optimal angular sensor separation for AOA localization," Signal Process., vol. 88, no. 5, pp. 1248-1260, 2008.

[20] R. Sim and N. Roy, "Global a-optimal robot exploration in SLAM," in Proceedings of the IEEE International Conference on Robotics and Automation (ICRA), Barcelona, Spain, 2005, pp. 661-666.

[21] T. Vidal-Calleja, A. Davison, J. Andrade-Cetto, and D. Murray, "Active control for single camera SLAM," in IEEE Int Conf on Robotics and Automation, Orlando, May 2006, 2006, pp. 1930-1936.

[22] Teledyne RD Instruments, "Explorer DVL," World Wide Web electronic publication, 2008. [Online]. Available: http://www.rdinstruments.com/explorer.html

[23] R. M. Eustice, O. Pizarro, and H. Singh, "Visually augmented navigation for autonomous underwater vehicles," IEEE Journal of Oceanic Engineering, vol. 33, no. 2, pp. 103-122, Apr. 2008. 